\title{
Spatial splay states in coupled map lattices and Josephson junction arrays
}

\author{
JOYDEEP SINGHA ${ }^{1, *}$, VALERIE TCHUYANG ${ }^{2}$, PATRICK LOUODOP ${ }^{2,3}$, ROBERT TCHITNGA $^{2}$, \\ HILDA A. CERDEIRA ${ }^{3}$ and NEELIMA GUPTE ${ }^{1}$ \\ ${ }^{1}$ Department of Physics, Indian Institute of Technology Madras, Chennai 600036, India \\ 2 Department of Physics, University of Dschang Cameroon \\ ${ }^{3}$ São Paulo State University (UNESP), Instituto de Física Teórica, Rua Dr. Bento Teobaldo Ferraz 271, Bloco II, \\ 01140-070 São Paulo, Brazil \\ ${ }^{*}$ Corresponding author. E-mail: joydeep@physics.iitm.ac.in
}

\begin{abstract}
We show the existence and stability of frozen splay states as well as temporally chaotic splay states in a coupled sine circle map lattice system using analytic and numerical techniques. The splay states are observed for very low values of the nonlinearity parameter, i.e., for circle maps which deviate very slightly from the shift map case. We also observe that, depending on the parameters of the system, the splay states bifurcate to mixed or chimera splay states, consisting of a mixture of splay and synchronised states, together with kinks in the phases of some of the maps and then to a globally synchronised state. We estimate the parameter regions where these pure states and the mixed states are seen. We also briefly show that similar spatial splay structures can exist in experimentally realisable systems like Josephson junction arrays and Hartley-like oscillator arrays.
\end{abstract}

Keywords. Splay states; chimera states; coupled map lattice.

PACS Nos 12.60.Jv; 12.10.Dm; 98.80.Cq; 11.30.Hv

\section{Introduction}

Spatially extended systems such as coupled map lattices (CML-s) show a rich variety of complex behaviours [1-4]. They support complex spatial structures whose dynamics and stability depend on the coupling scheme and coupling strength between the individual dynamical units, initial conditions as well as the size of the system. Some of the dynamical behaviours that are widely studied in such systems are synchronisation, spatio-temporal intermittency, chimera states, splay states and many more. Here we discuss the existence and stability of the splay states which are commonly seen in coupled phase oscillator systems. Let $\theta(0), \theta(1), \theta(2), \cdots, \theta(N-1)$ be the phases of $N$ such coupled oscillators in a system which is discrete in space and continuous in time. As mentioned in Refs [4-8], if the phases of the oscillators were to maintain a splay state having a temporal period $T$ then the phase of any $k$ th oscillator at any given time $t$ is given by $\theta_{k}(t)=\theta_{0}\left(t+\frac{T k}{N}\right)$, i.e., the oscillators are mutually phase shifted. The nature of this splay state has been studied in experimental systems like coupled Josephson junction arrays, coupled multi-mode laser systems, globally pulse-coupled rotators, coupled Stuart-Landau oscillators [4-6, 8,9]. The significance of this pure splay state in general was discussed in the context of beam steering and beam forming using repulsively coupled Stuart-Landau oscillators $[10,11]$. Here we study the splay state in a coupled map lattice (CML), a system which is discrete in both space and time. In this system we use a variant of the definition of splay states, where the phase of any $k$ th map at any time step is given by $\theta(k)=\theta(0)+2 \pi k / N$, where $k=0, \cdots, N-1$ [12-14]. This definition of the splay phase configuration implies that the phases of the maps at all time steps when plotted around a unit circle are always equally spaced irrespective of its temporal behavior. A snapshot of this phase configuration is shown in figure $2 \mathrm{a}$ where two consecutive sites maintain the above restriction on their phases for $\theta(0)=0$, with their labels, $0,1,2, \cdots, 2 N$ for $2 N$ sites, plotted along the $x$-axis. We use the term 


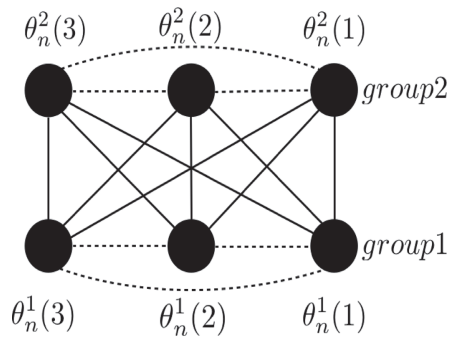

(a)

Figure 1. This illustrates the connection scheme of the globally connected network. The black dots represent the maps in each group with 3 maps in each group. Each map in the system is coupled to the maps in its own group via a coupling $\epsilon_{1}$ (represented by dotted edges) and to the maps in the other groups via a coupling $\epsilon_{2}$ (represented by solid edges).

'spatial splay states' to distinguish it from the former definition.

We show here that a CML having two populations of identical sine circle maps sharing an intra-population coupling as well as an inter-population coupling (see figure 1) can support a pure spatial splay state for small nonlinearity. We analyse their stability by using numerical and analytical techniques and find that the pure spatial splay states are chaotic in our CML. We explore their bifurcation route as the nonlinearity in the system is increased. We also show that the spatial splay structures can also be seen in an array of Josephson junctions with a similar coupling scheme as in the CML, as well as in a Hartley-like oscillator array via a nearest neighbour coupling.

\section{The coupled map lattice model}

We use a bipartite coupled map lattice with two groups of identical sine circle maps, in which all the circle maps in a group are coupled to each other with a given coupling strength and to all the maps in the other group with a different coupling strength [1]. The equation for a single sine circle map is given by

$\theta_{n+1}=\theta_{n}+\Omega-\frac{K}{2 \pi} \sin \left(2 \pi \theta_{n}\right) \quad \bmod 1$,

where $\theta_{n}$ is the phase at the $n$th time step and $\Omega$ and $K$ are respectively the frequency ratio and nonlinearity parameters. A single sine circle map shows mode locking structures organized by frequency locking and quasi-periodic behaviour. Chaos appears via period doubling and quasi-periodicity depending on the parameters $K$ and $\Omega[15,16]$. Using eq. (1), we write the evolution equation for the phase $\theta$ of the $i$ th coupled sine circle map of our CML as

$$
\begin{aligned}
\theta_{n+1}^{\sigma}(i) & =\theta_{n}^{\sigma}(i)+\Omega-\frac{K}{2 \pi} \sin \left(2 \pi \theta_{n}^{\sigma}(i)\right)+\sum_{\sigma^{\prime}=1}^{2} \frac{\epsilon_{\sigma \sigma^{\prime}}}{N_{\sigma^{\prime}}} \\
& \times\left[\sum_{j=1}^{N_{\sigma^{\prime}}}\left(\theta_{n}^{\sigma^{\prime}}(i)+\Omega-\frac{K}{2 \pi} \sin \left(2 \pi \theta_{n}^{\sigma^{\prime}}(i)\right)\right)\right] \bmod 1 .
\end{aligned}
$$

Here $\sigma$ takes values 1,2, since the CML under consideration has two groups and the number of maps in the group $\sigma$ is given by $N_{\sigma}$ (a simple schematic of the CML with $N=3$ is shown in figure 1).

The coupling strength parameters are defined as $\epsilon_{11}=\epsilon_{22}=\epsilon_{1}$ and $\epsilon_{12}=\epsilon_{21}=\epsilon_{2}$, where $\epsilon_{1}+\epsilon_{2}=1$. Thus our CML in eq. (2) is controlled by three independent parameters $K, \Omega, \epsilon_{1}$. We restrict these three parameters to lie within $[0,1]$. To show the existence of the spatial splay states we use a unique initial condition, namely a system-wide single spatial splay state consisting of all the $2 N$ lattice sites where the phase difference between any two consecutive lattice sites is given by $1 / 2 N$ (see figure $2 a$ ). We identify the parameter values where this single spatial splay state can be frozen in time. We show that this frozen state is unstable. At low values of $K$ $\left(\approx 10^{-10}\right)$, if eq. $(2)$ evolves from this initial condition, then the single spatial splay state breaks into two identical splay states, each of which spans each of the groups. We explore the stability and bifurcation of the two copy splay states and also discuss the phase diagram in the $\Omega-\epsilon_{1}$ space.

\subsection{Frozen spatial splay states}

Let the phases of the maps for a single splay state spanning the $2 N$ lattice sites be $\theta_{s}^{1}(0)=0, \theta_{s}^{1}(1)=\frac{1}{2 N}, \theta_{s}^{1}(2)$ $=\frac{2}{2 N}, \cdots, \theta_{s}^{1}(N-1)=\frac{N-1}{2 N}, \theta_{s}^{2}(0)=\frac{N}{2 N}, \cdots, \theta_{s}^{2}(N-1)$ $=\frac{2 N-1}{2 N}$. For these phases to be frozen in time we must have

$$
\begin{aligned}
& \frac{K}{2 \pi} \sin \left(2 \pi \theta_{s}^{\sigma}(i)\right)= \\
& \Omega+\sum_{\sigma^{\prime}=1}^{2} \frac{\epsilon_{\sigma \sigma^{\prime}}}{N_{\sigma^{\prime}}}\left[\sum_{j=1}^{N_{\sigma^{\prime}}}\left(\theta_{s}^{\sigma^{\prime}}+\Omega-\frac{K}{2 \pi} \sin \left(2 \pi \theta_{s}^{\sigma^{\prime}}(i)\right)\right)\right]-q_{i}^{\sigma},
\end{aligned}
$$

where $q_{i}^{\sigma}$ is an integer. For any two maps $i, j$ in a given group $\sigma$ we can write

$\frac{K}{2 \pi}\left(\sin 2 \pi \theta_{s}^{\sigma}(i)-\sin 2 \pi \theta_{s}^{\sigma}(j)\right)=q_{j}^{\sigma}-q_{i}^{\sigma}$. 


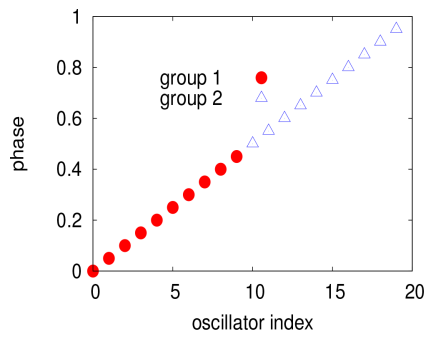

(a)

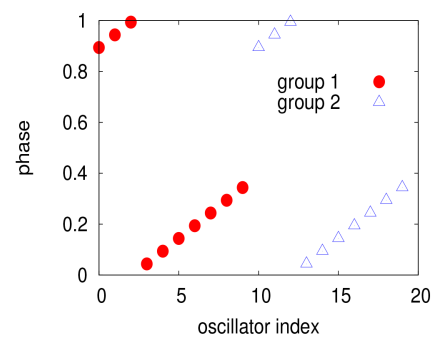

(b)

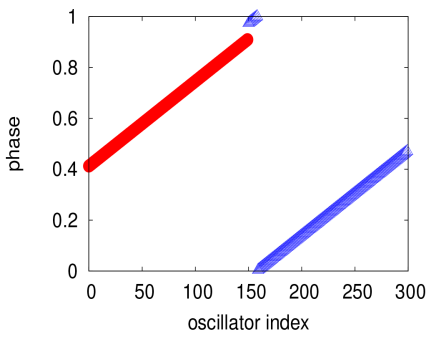

(c)

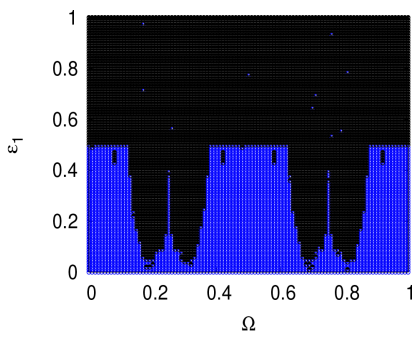

(d)

Figure 2. (a) Single spatial splay state throughout the system where the difference between the phases of two consecutive maps is $\frac{1}{20}$, where the system has 20 maps, with 10 maps in each group. (b) When $K=10^{-10}, \Omega=\frac{2}{7}, \epsilon_{1}=0.01$, eq. (2) evolves to a spatial splay state with two copies. The phase difference between any two consecutive maps of each copy is given by $\frac{1}{20}$. The phase difference between the last map of group 1 and the first map of group 2 is .55. (c) Two splay states with a phase slip between group 1 and group 2 are shown for parameter values $K=0, \Omega=0.04, \epsilon_{1}=.67, N=150$. (d) The phase diagram in $\Omega-\epsilon_{1}$ space obtained with 150 maps in each group. Blue circles denote the two copy splay states and black circles denote two splay states with a phase slip.

The left hand side of the above equation can take values between $\left[-\frac{1}{\pi}, \frac{1}{\pi}\right]$. As $q_{i}^{\sigma}$ s are integers, $q_{j}^{\sigma}-q_{i}^{\sigma}$ can only be zero. So the frozen single spatial splay state can exist only for $K=0$. If we substitute the phases and use $K=0$ in eq. (2) we find that the single spatial splay state can be frozen in time when the parameters are $K=0, \epsilon_{1}=0.5, \Omega=0.5-\frac{2 N-1}{8 N}, 1-\frac{2 N-1}{8 N}$ for a given $N$.

\subsection{Stability analysis}

As mentioned in Refs $[1,18]$ the general form of the Jacobian for the CML is obtained after linearizing eq. (2) and is given as

$J=\left[\begin{array}{ll}A & B \\ C & D\end{array}\right]$.

Here, $A, B, C, D$ are matrices of order $N \times N$ which are as follows

$$
\begin{gathered}
A=\left[\begin{array}{cccc}
\left(2-\frac{N-1}{N} \epsilon_{1}-\epsilon_{2}\right) f^{\prime}\left(\theta_{n}^{1}(1)\right) & \frac{\epsilon_{1}}{N} f^{\prime}\left(\theta_{n}^{1}(2)\right) & \cdots & \frac{\epsilon_{1}}{N} f^{\prime}\left(\theta_{n}^{1}(N)\right) \\
\frac{\epsilon_{1}}{N} f^{\prime}\left(\theta_{n}^{1}(1)\right) & \left(2-\frac{N-1}{N} \epsilon_{1}-\epsilon_{2}\right) f^{\prime}\left(\theta_{n}^{1}(2)\right) & \cdots & \frac{\epsilon_{1}}{N} f^{\prime}\left(\theta_{n}^{1}(N)\right) \\
\cdots & \cdots & \cdots & \cdots \\
\frac{\epsilon_{1}}{N} f^{\prime}\left(\theta_{N}^{1}(1)\right) & \frac{\epsilon_{1}}{N} f^{\prime}\left(\theta_{N}^{1}(2)\right) & \cdots & \left(2-\frac{N-1}{N} \epsilon_{1}-\epsilon_{2}\right) f^{\prime}\left(\theta_{N}^{1}(N)\right)
\end{array}\right] \\
D=\left[\begin{array}{cccc}
\left(2-\frac{N-1}{N} \epsilon_{1}-\epsilon_{2}\right) g^{\prime}\left(\theta_{n}^{2}(1)\right) & \frac{\epsilon_{1}}{N} g^{\prime}\left(\theta_{n}^{2}(2)\right) & \cdots & \frac{\epsilon_{1}}{N} g^{\prime}\left(\theta_{n}^{2}(N)\right) \\
\frac{\epsilon_{1}}{N} g^{\prime}\left(\theta_{n}^{2}(1)\right) & \left(2-\frac{N-1}{N} \epsilon_{1}-\epsilon_{2}\right) g^{\prime}\left(\theta_{n}^{2}(2)\right) & \cdots & \frac{\epsilon_{1}}{N} g^{\prime}\left(\theta_{n}^{2}(N)\right) \\
\cdots & \cdots & \cdots & \cdots \\
\frac{\epsilon_{1}}{N} g^{\prime}\left(\theta_{N}^{2}(1)\right) & \frac{\epsilon_{1}}{N} g^{\prime}\left(\theta_{N}^{2}(2)\right) & \cdots & \left(2-\frac{N-1}{N} \epsilon_{1}-\epsilon_{2}\right) g^{\prime}\left(\theta_{N}^{2}(N)\right)
\end{array}\right]
\end{gathered}
$$

$$
B=\left[\begin{array}{cccc}
\frac{\epsilon_{2}}{N} g^{\prime}\left(\theta_{n}^{2}(1)\right) & \frac{\epsilon_{2}}{N} g^{\prime}\left(\theta_{n}^{2}(2)\right) & \ldots & \frac{\epsilon_{2}}{N} g^{\prime}\left(\theta_{n}^{2}(N)\right) \\
\frac{\epsilon_{2}}{N} g^{\prime}\left(\theta_{n}^{2}(1)\right) & \frac{\epsilon_{2}}{N} g^{\prime}\left(\theta_{n}^{2}(2)\right) & \ldots & \frac{\epsilon_{2}}{N} g^{\prime}\left(\theta_{n}^{2}(N)\right) \\
\ldots & \ldots & \ldots & \ldots \\
\frac{\epsilon_{2}}{N} g^{\prime}\left(\theta_{n}^{2}(1)\right) & \frac{\epsilon_{2}}{N} g^{\prime}\left(\theta_{n}^{2}(2)\right) & \ldots & \frac{\epsilon_{2}}{N} g^{\prime}\left(\theta_{n}^{2}(N)\right)
\end{array}\right] \quad(8) \quad C=\left[\begin{array}{cccc}
\frac{\epsilon_{2}}{N} f^{\prime}\left(\theta_{n}^{2}(1)\right) & \frac{\epsilon_{2}}{N} f^{\prime}\left(\theta_{n}^{2}(2)\right) & \ldots & \frac{\epsilon_{2}}{N} f^{\prime}\left(\theta_{n}^{2}(N)\right) \\
\frac{\epsilon_{2}}{N} f^{\prime}\left(\theta_{n}^{2}(1)\right) & \frac{\epsilon_{2}}{N} f^{\prime}\left(\theta_{n}^{2}(2)\right) & \ldots & \frac{\epsilon_{2}}{N} f^{\prime}\left(\theta_{n}^{2}(N)\right) \\
\ldots & \ldots & \ldots & \ldots \\
\frac{\epsilon_{2}}{N} f^{\prime}\left(\theta_{n}^{2}(1)\right) & \frac{\epsilon_{2}}{N} f^{\prime}\left(\theta_{n}^{2}(2)\right) & \ldots & \frac{\epsilon_{2}}{N} f^{\prime}\left(\theta_{n}^{2}(N)\right)
\end{array}\right],
$$


where $f^{\prime}\left(\theta_{n}^{1}(j)\right)=1-K \cos \left(2 \pi \theta_{N}^{1}(j)\right)$ and $g^{\prime}\left(\theta_{n}^{2}(j)\right)$ $=1-K \cos \left(2 \pi \theta_{N}^{2}(j)\right)$. Using $K=0$, we obtain the Jacobian $\left(\widetilde{J}_{S}\right)$ of the phases of the single spatial splay state.

$\widetilde{J}_{S}=\left[\begin{array}{ll}A & B \\ B & A\end{array}\right]$,

where

$A=\left[\begin{array}{cccc}\left(1+\frac{\epsilon_{1}}{N}\right) & \frac{\epsilon_{1}}{N} & \cdots & \frac{\epsilon_{1}}{N} \\ \frac{\epsilon_{1}}{N} & \left(1+\frac{\epsilon_{1}}{N}\right) & \cdots & \frac{\epsilon_{1}}{N} \\ \cdots & \cdots & \cdots & \cdots \\ \frac{\epsilon_{1}}{N} & \frac{\epsilon_{1}}{N} & \cdots & \left(1+\frac{\epsilon_{1}}{N}\right)\end{array}\right]$

$B=\left[\begin{array}{cccc}\frac{\epsilon_{2}}{N} & \frac{\epsilon_{2}}{N} & \cdots & \frac{\epsilon_{2}}{N} \\ \frac{\epsilon_{2}}{N} & \frac{\epsilon_{2}}{N} & \cdots & \frac{\epsilon_{2}}{N} \\ \cdots & \cdots & \cdots & \cdots \\ \frac{\epsilon_{2}}{N} & \frac{\epsilon_{2}}{N} & \cdots & \frac{\epsilon_{2}}{N}\end{array}\right]$.

$\widetilde{J}_{S}$ is a block circulant matrix which can be block diagonalized [17] using $P=F_{2} \otimes N$, where $F_{2}$ is a $2 \times 2$ Fourier matrix and $I_{N}$ is an identity matrix of order $N \times N$. So we have

$\mathbf{P}^{-1} \widetilde{J}_{S} \mathbf{P}=\widetilde{J}_{S}^{*}=\left[\begin{array}{cc}A+B & 0 \\ 0 & A-B\end{array}\right]$,

where

$A+B=\left[\begin{array}{cccc}\left(1+\frac{\epsilon_{1}+\epsilon_{2}}{N}\right) & \frac{\epsilon_{1}+\epsilon_{2}}{N} & \cdots & \frac{\epsilon_{1}+\epsilon_{2}}{N} \\ \frac{\epsilon_{1}+\epsilon_{2}}{N} & \left(1+\frac{\epsilon_{1}+\epsilon_{2}}{N}\right) & \cdots & \frac{\epsilon_{1}+\epsilon_{2}}{N} \\ \cdots & \cdots & \cdots & \cdots \\ \frac{\epsilon_{1}+\epsilon_{2}}{N} & \frac{\epsilon_{1}+\epsilon_{2}}{N} & \cdots & \left(1+\frac{\epsilon_{1}+\epsilon_{2}}{N}\right)\end{array}\right]$
$A-B=\left[\begin{array}{cccc}\left(1+\frac{\epsilon_{1}-\epsilon_{2}}{N}\right) & \frac{\epsilon_{1}-\epsilon_{2}}{N} & \cdots & \frac{\epsilon_{1}-\epsilon_{2}}{N} \\ \frac{\epsilon_{1}-\epsilon_{2}}{N} & \left(1+\frac{\epsilon_{1}-\epsilon_{2}}{N}\right) & \cdots & \frac{\epsilon_{1}-\epsilon_{2}}{N} \\ \cdots & \cdots & \cdots & \cdots \\ \frac{\epsilon_{1}-\epsilon_{2}}{N} & \frac{\epsilon_{1}-\epsilon_{2}}{N} & \cdots & \left(1+\frac{\epsilon_{1}-\epsilon_{2}}{N}\right)\end{array}\right]$.

The matrix $\widetilde{J}_{S}$ has eigenvalues $2,2 \epsilon_{1}$, and $2 N-2$ fold degenerate eigenvalues 1 since $A+B$ and $A-B$ are circulant matrices. Using $\epsilon=0.5$ we conclude that the frozen single spatial splay state is unstable in one direction, and neutrally stable in all the other directions. However, using the single spatial splay state as an initial condition for $K=0$, eq. (2) always evolves to either two copy splay states (figure 2b) or two copy splay states with phase slips (figure $2 \mathrm{c}$ ). Figure $2 \mathrm{~d}$ shows the phase diagram in the $\Omega-\epsilon_{1}$ parameter space at $K=10^{-8}$ and $N$ $=150$. We divide the parameter space into a $100 \times 100$ grid and eq. (2) is evolved for $1.5 \times 10^{6}$ time steps from single spatial splay state, at each grid point. The two copy splay states occupy $32.11 \%$ of the phase diagram. At the boundary of this region the two copy splay states bifurcate to the phase slipped splay states due to the change in parameters.

\subsection{Stability analysis of two copy splay states}

We note that two copy splay states consist of two identical splay states constituted separately by the maps in each group. Let us write the phases of the maps in any of them at a time step $n$ as $\theta(0), \theta(1), \theta(2), \cdots, \theta(N-1)$, where $\theta(i)-\theta(i+1)=\frac{1}{2 N}$. For these phases, the Jacobian of the CML [1] becomes

$\widetilde{J}_{2 S}=\left[\begin{array}{ll}C & D \\ D & C\end{array}\right]$,

where

$C=\left[\begin{array}{cccc}\left(1+\frac{\epsilon_{1}}{N}\right)(1-K \cos 2 \pi \theta(0)) & \frac{\epsilon_{1}}{N}(1-K \cos 2 \pi \theta(1)) & \cdots & \frac{\epsilon_{1}}{N}(1-K \cos 2 \pi \theta(N-1)) \\ \frac{\epsilon_{1}}{N}(1-K \cos 2 \pi \theta(0)) & \left(1+\frac{\epsilon_{1}}{N}\right)(1-K \cos 2 \pi \theta(1)) & \cdots & \frac{\epsilon_{1}}{N}(1-K \cos 2 \pi \theta(N-1)) \\ \cdots & \cdots & \cdots & \cdots \\ \frac{\epsilon_{1}}{N}(1-K \cos 2 \pi \theta(0)) & \frac{\epsilon_{1}}{N}(1-K \cos 2 \pi \theta(1)) & \cdots & \left(1+\frac{\epsilon_{1}}{N}\right)(1+K \cos 2 \pi \theta(N-1))\end{array}\right]$

$D=\left[\begin{array}{cccc}\frac{\epsilon_{2}}{N}(1-K \cos 2 \pi \theta(0)) & \frac{\epsilon_{2}}{N}(1-K \cos 2 \pi \theta(1)) & \cdots & \frac{\epsilon_{2}}{N}(1-K \cos 2 \pi \theta(N-1)) \\ \left.\frac{\epsilon_{2}}{N}(1-K \cos 2 \pi \theta(0))\right) & \frac{\epsilon_{2}}{N}(1-K \cos 2 \pi \theta(1)) & \cdots & \frac{\epsilon_{2}}{N}(1-K \cos 2 \pi \theta(N-1)) \\ \cdots & \cdots & \cdots & \cdots \\ \frac{\epsilon_{2}}{N}(1-K \cos 2 \pi \theta(0)) & \frac{\epsilon_{2}}{N}(1-K \cos 2 \pi \theta(1)) & \cdots & \frac{\epsilon_{2}}{N}(1-K \cos 2 \pi \theta(N-1))\end{array}\right]$, 
where we use the general form of the Jacobian given in [1]. We numerically calculate the eigenvalues of this one step Jacobian matrix in eq. (16) for the two copy spatial splay states. Figure $3 \mathrm{a}$ shows that the largest eigenvalue is greater than one. This indicates that the two copy splay states are temporally unstable. We see that $\widetilde{J}_{2 S}$ is a block circulant matrix.
So it can be block diagonalised again by $P$. We have

$\mathbf{P}^{-1} \widetilde{J}_{2 S} \mathbf{P}=\widetilde{J}_{2 S}^{*}=\left[\begin{array}{cc}C+D & 0 \\ 0 & C-D\end{array}\right]$,

where

$C+D=\left[\begin{array}{cccc}\left(1+\frac{\epsilon_{1}+\epsilon_{2}}{N}\right)(1-K \cos 2 \pi \theta(0)) & \frac{\epsilon_{1}+\epsilon_{2}}{N}(1-K \cos 2 \pi \theta(1)) & \cdots & \frac{\epsilon_{1}+\epsilon_{2}}{N}(1-K \cos 2 \pi \theta(N-1)) \\ \frac{\epsilon_{1}+\epsilon_{2}}{N}(1-K \cos 2 \pi \theta(0)) & \left(1+\frac{\epsilon_{1}+\epsilon_{2}}{N}\right)(1-K \cos 2 \pi \theta(1)) & \cdots & \frac{\epsilon_{1}+\epsilon_{2}}{N}(1-K \cos 2 \pi \theta(N-1)) \\ \cdots & \ldots & \cdots & \ldots \\ \frac{\epsilon_{1}+\epsilon_{2}}{N}(1-K \cos 2 \pi \theta(0)) & \frac{\epsilon_{1}+\epsilon_{2}}{N}(1-K \cos 2 \pi \theta(1)) & \cdots & \left(1+\frac{\epsilon_{1}+\epsilon_{2}}{N}\right)(1-K \cos 2 \pi \theta(N-1))\end{array}\right]$

$C-D=\left[\begin{array}{cccc}\left(1+\frac{\epsilon_{1}-\epsilon_{2}}{N}\right)(1-K \cos 2 \pi \theta(0)) & \frac{\epsilon_{1}-\epsilon_{2}}{N}(1-K \cos 2 \pi \theta(1)) & \cdots & \frac{\epsilon_{1}-\epsilon_{2}}{N}(1-K \cos 2 \pi \theta(N-1)) \\ \frac{\epsilon_{1}-\epsilon_{2}}{N}(1-K \cos 2 \pi \theta(0)) & \left(1+\frac{\epsilon_{1}-\epsilon_{2}}{N}\right)(1-K \cos 2 \pi \theta(1)) & \cdots & \frac{\epsilon_{1}-\epsilon_{2}}{N}(1-K \cos 2 \pi \theta(N-1)) \\ \ldots & \ldots & \ldots & \ldots \\ \frac{\epsilon_{1}-\epsilon_{2}}{N}(1-K \cos 2 \pi \theta(0)) & \frac{\epsilon_{1}-\epsilon_{2}}{N}(1-K \cos 2 \pi \theta(1)) & \cdots & \left(1+\frac{\epsilon_{1}-\epsilon_{2}}{N}\right)(1-K \cos 2 \pi \theta(N-1))\end{array}\right]$.

As we have chosen $K$ to lie in the interval [0,1], $C+D$ becomes a non-negative irreducible matrix. Let $(C-D)^{+}$ be the matrix constructed by taking the modulus of each of the elements in $C-D$. It is easy to see that each element of $(C+D)$ is greater than or equal to the corresponding element of the matrix $(C-D)^{+}$. Then according to Frobenious-Perron theorem and Wielandt's lemma [17], the largest eigenvalue of the Jacobian for the two copy splay state is the largest eigenvalue $\lambda$ of the block matrix $C+D$. Using the Gershgorin theorem [18] the

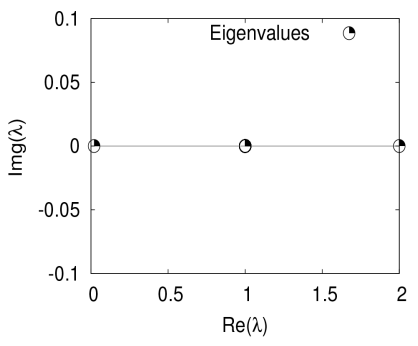

(a)

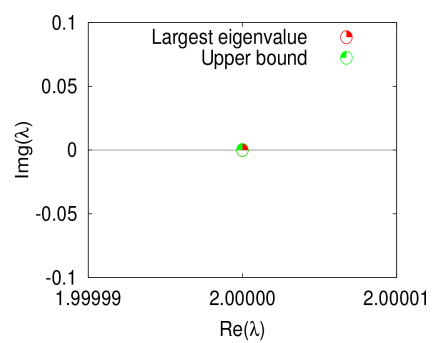

(b)
Figure 3. (a) The numerically calculated eigenvalues of $\widetilde{J}_{2 S}$ for a system with 150 lattice sites in each group. The parameters are $K=10^{-10}, \Omega=2 / 7, \epsilon_{1}=0.01$. (b) The location of the upper bound and the largest eigenvalue are shown. These coincide within graphical accuracy. upper bound on $\lambda$ is found to be

$$
\min \left(\max \left(c_{j}+r_{j}\right), \max \left(c_{i}+s_{i}\right)\right),
$$

where

$$
c_{j}=\left(1+\frac{\epsilon_{1}+\epsilon_{2}}{N}\right)\left(1-K \cos \left(2 \pi \theta_{0}+\frac{2 \pi j}{2 N}\right)\right)
$$

$$
\begin{aligned}
& r_{j}=\frac{N-1}{N} \\
& -\frac{K}{N}\left[\frac{\cos (2 \pi \theta(0))\left(1-\cos \frac{\pi}{N}\right)-\sin (2 \pi \theta(0)) \sin \left(\frac{\pi}{N}\right)}{1-\cos \left(\frac{\pi}{N}\right)}\right] \\
& +\frac{K}{N} \cos \left(2 \pi \theta(0)+\frac{\pi j}{N}\right) \\
& s_{i}=\frac{N-1}{N}\left(1-K \cos \left(2 \pi \theta(0)+\frac{\pi i}{N}\right)\right),
\end{aligned}
$$

with $1 \leq j \leq N$ and $1 \leq i \leq N$ (see [18] and the references therein for details) The largest eigenvalue obtained numerically by diagonalising the matrix $\widetilde{J}_{2 S}$ is shown in figure $3 \mathrm{a}$. Figure $3 \mathrm{~b}$ shows that the largest eigenvalue saturates the bound here. 


\subsection{Temporal behavior and bifurcations}

2.4.1 Lyapunov exponent and order parameter. We showed that the numerically calculated largest eigenvalue for the two copy splay states exceeds one. To see if the splay states maintain their spatial structure we calculate the order parameter $R=|\langle\exp (i 2 \pi \theta)\rangle|$, where the average is taken over all the maps with the same dynamics at each time step. We calculate this quantity by taking the average over the whole system, as we have two synchronising splay copies. Figure 4a shows that $R$ is constant which implies that the phase distribution of the maps for the two copy splay states is stable. To verify if this state is temporally chaotic we calculate the largest Lyapunov exponent, using the Gram-Schmidt procedure for $10^{6}$ time steps, after discarding the initial $3 \times 10^{6}$ time steps. The largest Lyapunov exponent turns out to be 0.693 (see figure $4 b$ ) which indicates that the two copy splay states are temporally chaotic.

2.4.2 Bifurcations. We list the states that can be observed as $K$ is increased (see figure 5) when eq. (2) is evolved from single spatial splay state as initial condition. (a) The two copy splay states appear in the range $0<K<10^{-7}$ (figure 5a). (b) Like the two copy splay state, the phase of the $i$ th map and the $(i+N)$ th map are equal where $N$ is the number of maps in each group, although the difference between the consecutive maps increases as $i$ increases in a group (figure 5b). This appears approximately in the range $10^{-6}<K<10^{-5}$. (c) If we increase $K$ to $10^{-2}$ we see the splay chimera structure where all the maps in group one are spatially synchronised and maps at sites 150 to 200 and 250 to 300 show splay-like diagonal structure and the sites 200 to 250 show a phase jump (figure $5 \mathrm{c}$ ). We observe this type of spatial behaviour in the range $10^{-4}<K<.05$.

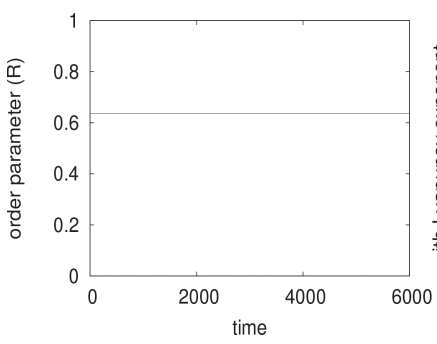

(a)

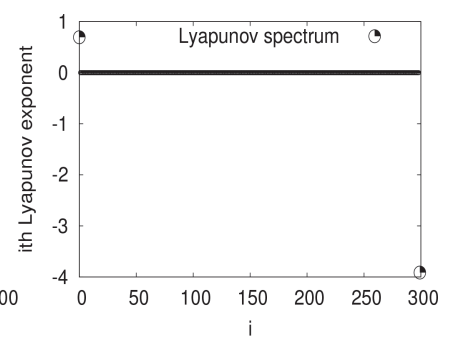

(b)
Figure 4. (a) The variation of the order parameter with time for 6000 steps. The figure is plotted after discarding the initial $3 \times 10^{6}$ time steps. (b) The Lyapunov spectrum at $K=10^{-10}, \Omega=2 / 7, \epsilon_{1}$ $=0.01, N=150$ for the two copy splay states. The two circles denote the largest and the smallest Lyapunov exponent and the smaller sized points are those which are very near to zero.

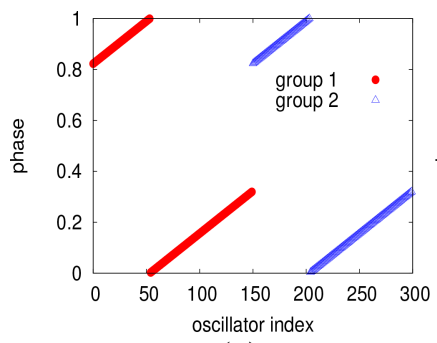

(a)

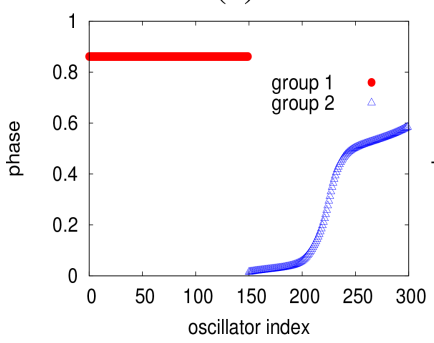

(c)

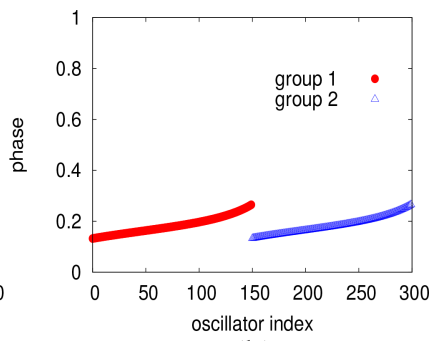

(b)

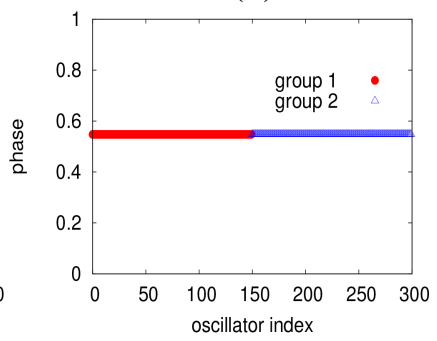

(d)
Figure 5. (a) The initial condition where all the maps constitute a spatial splay state was used in each case. The snapshots are taken after 3,000,000 time steps The parameters are $N=150, \Omega=\frac{2}{7}, \epsilon_{1}=0.01$. Splay states were obtained at parameter values $K=10^{-11}$. (b) For the parameter values $K=10^{-6}$, the two groups still synchronise but the size of the splay states decrease. (c) The splay chimera state is obtained for $K=10^{-2}$. (d) Increasing $K$ to 1 gives a global synchronised state.

(d) The whole system is spatially synchronised for $K$ above .05 (figure $5 \mathrm{~d}$ ). The stability of each of these structures w.r.t. the variation of $K$ can be qualitatively
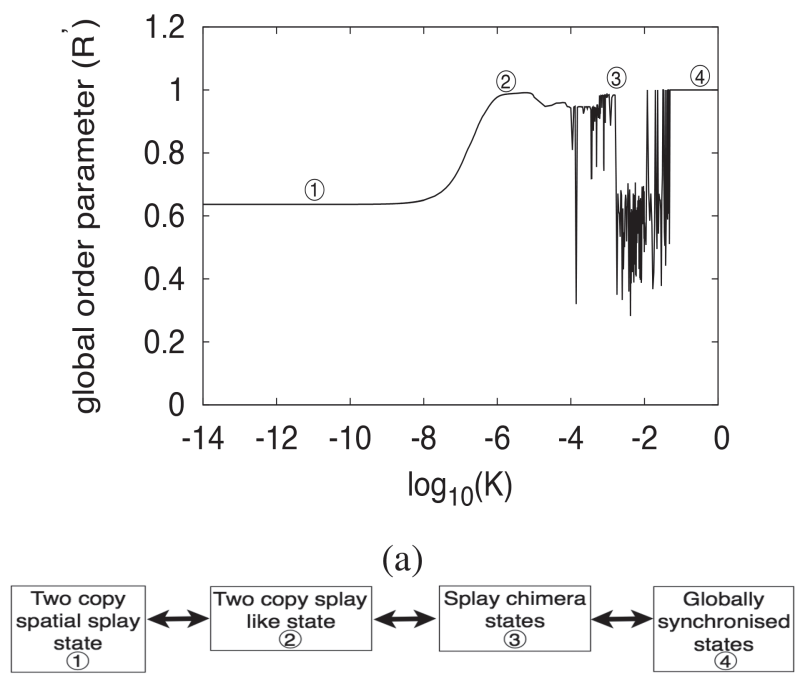

(b)

Figure 6. (a) The order parameter is calculated for values of $K$ between zero to one. For each value of $K$, eq. (2) is iterated for $3 \times 10^{6}$ times after which the order parameter for the whole system is calculated. (b) A schematic of the bifurcation route for $\epsilon_{1}=0.01$ and $\Omega=\frac{2}{7}$ and varying $K$ from 0 to 1 . 


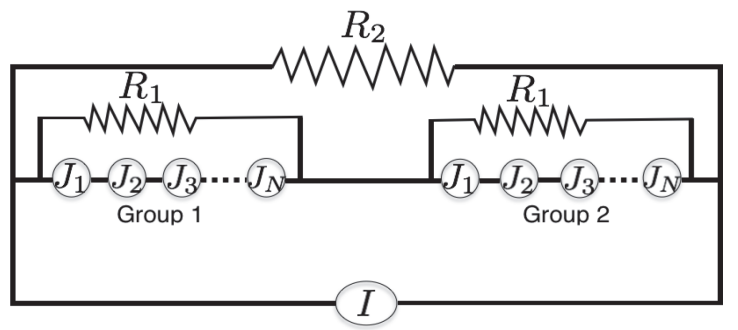

Figure 7. A schematic of the two array Josephson junctions. $J_{i} \mathrm{~s}$ are the junctions. $I$ is the bias current.

understood by looking at the global order parameter $R^{\prime}=\sum_{i=1}^{2 N} e^{i 2 \pi \theta} / 2 N$ (see figure 6a). The variation in $R^{\prime}$ in the range $10^{-4}<K<.05$ indicates a variation in the splay chimera structures. It also shows that the spatial structures of the two copy splay states, splay states with phase kinks and the globally synchronised state, are maintained with the variation of $K$. A schematic (see figure $6 \mathrm{~b}$ ) of this bifurcation route is given for convenience.

We now show the existence of splay states in globally coupled Josephson junctions and in an array of Hartleylike oscillators with nearest neighbour coupling.

\section{Spatial splay states in globally coupled Josephson junction array}

Let us consider two arrays of Josephson junctions consisting of $N$ identical junctions in each array which are coupled using the resistors $R_{1}, R_{2}$ as shown in figure 7 . We assume that these $2 N$ junctions are overdamped [5]. Applying Kirchoff's circuit law, we can write the governing equations as

$$
\begin{aligned}
I_{b}= & I_{c} \sin \phi_{i}^{\sigma}+\frac{\hbar}{2 e R} \dot{\phi}_{i}^{\sigma}+\frac{\hbar}{2 e R_{1}} \sum_{i=1}^{N} \dot{\phi}_{i}^{\sigma} \\
& +\frac{\hbar}{2 e R_{2}} \sum_{\sigma=1}^{2}\left(\sum_{i=1}^{N} \dot{\phi}_{i}^{\sigma}\right),
\end{aligned}
$$

where $\sigma$ denotes the group and takes values $1,2 . \phi_{i}^{\sigma}$ is the quantum phase difference over the $i$ th junction in the group $\sigma, R$ is the junction resistance, $I_{c}$ is the critical current, $\hbar$ is the Planck's constant and $e$ is the electron charge. Introducing the dimensionless time $\tau=\frac{2 e R I_{c}}{\hbar}$ and dividing eq. (25) by $I_{c}$ we obtain

$\frac{I}{I_{c}}=\sin \phi_{i}^{\sigma}+\phi_{i}^{\prime \sigma}+\frac{R}{R_{1}} \sum_{i=1}^{N} \phi_{i}^{\prime \sigma}+\frac{R}{R_{2}}\left(\sum_{i=1}^{N} \phi_{i}^{\prime 1}+\sum_{i=1}^{N} \phi_{i}^{\prime 2}\right)$.

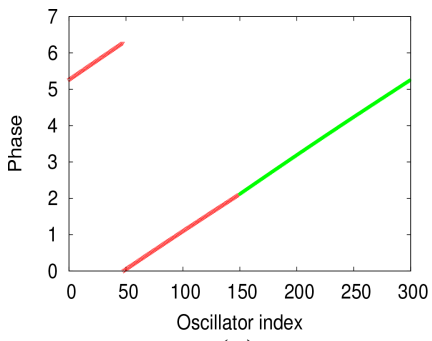

(a)

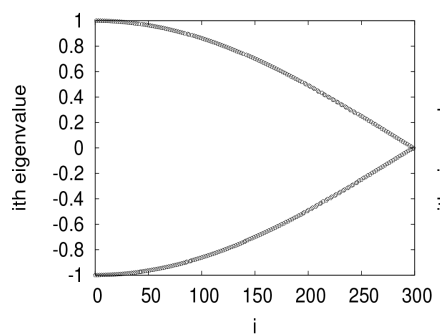

(c)

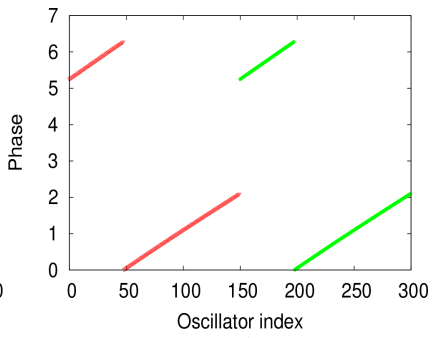

(b)

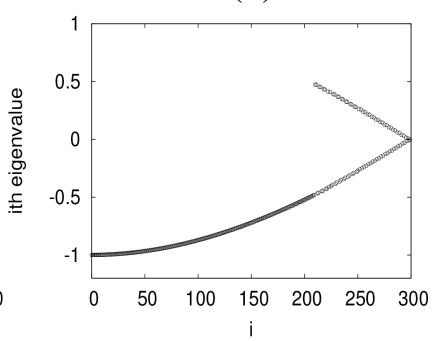

(d)
Figure 8. (a) Single spatial splay state and (b) two copy splay state. The parameters are $I / I_{c}=10^{6}, R / R_{1}=0.01, R / R_{2}=0.93$, $N=150$ for both the cases. The initial condition for (a) is a single spatial splay configuration and for (b) is a two copy splay configuration. The snapshots are taken after 1000 seconds in dimensionless time units. (c) and (d) show the eigenvalues for the one step Jacobian matrix for (a) and (b) respectively.

If we sum eq. (26), then we obtain the equation for the rate of change of the quantum phase difference of the $i$ th junction in group $\sigma$. Thus we have

$$
\begin{gathered}
\phi_{i}^{\prime \sigma}=\frac{I}{I_{c}(A+B)}-\sin \phi_{i}^{\sigma}+\left(\frac{B}{N\left(A^{2}-B^{2}\right)}\right) \sum_{i=1}^{N} \sin \phi_{i}^{\sigma^{\prime}} \\
+\left(\frac{1}{N}-\frac{A}{N\left(A^{2}-B^{2}\right)}\right) \sum_{i=1}^{N} \sin \phi_{i}^{\sigma},
\end{gathered}
$$

where $A=1+\frac{R N}{R_{1}}+\frac{R N}{R_{2}}, B=\frac{R N}{R_{2}} \cdot \sigma^{\prime}$ also denotes the group and $\sigma^{\prime} \neq \sigma$. So the circuit parameters are $\frac{I}{I_{c}}, \frac{R}{R_{1}}, \frac{R}{R_{2}}$. We find both the single spatial splay configuration as well as the two copy splay state in this system (see figure $8 \mathrm{a}$ and $\mathrm{b}$ ). Figure $8 \mathrm{c}$ shows that the single spatial splay state has $N$ number of pairwise locally stable and unstable directions while there exist local stable directions in the case of two copy splay state (figure 8d).

Splay states can also be seen in yet another practical context.

\section{Splay states in the locally coupled Josephson junction-based Hartley-like oscillator array}

We consider a circuit consisting of a Josephson junction with a transformer as shown in figure 9. The internal 


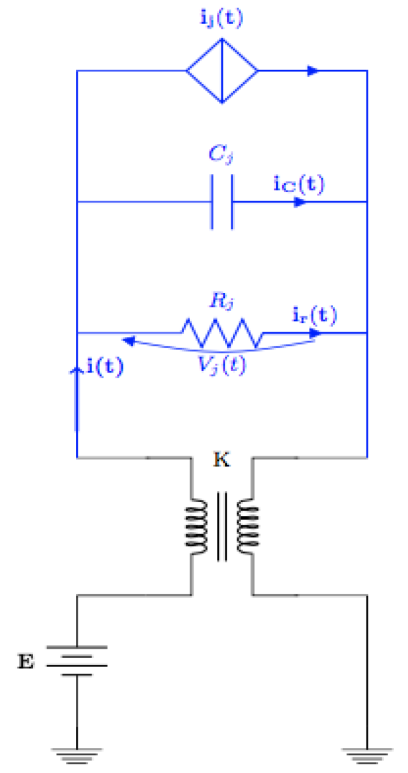

Figure 9. A schematic of the Hartley-like oscillator using a Josephson junction. The equivalent circuit of a single Josephson junction is drawn in blue.

capacitance of the Josephson junction plays the role of the capacitor, necessary for the Hartley scheme. Applying Kirchhoff's laws to the circuit we can obtain the following set of equations

$$
\begin{aligned}
\dot{\phi}(t) & =V_{j}(t) \\
\dot{V}_{j}(t) & =\frac{1}{C_{j}}\left(I_{j}(t)-\sin (\phi(t))-\frac{V_{j}(t)}{R_{j}}\right) \\
\dot{I}_{j}(t) & =\frac{1}{L_{1}+L_{2}+2 K}\left(E-V_{j}(t)\right),
\end{aligned}
$$

where $L_{1}, L_{2}$ are the self inductances of the coils in the transformer and $K$ is the mutual inductance between the two coils. We also have $I(t)=I_{j}+I_{C}+I_{r}$. If we introduce $\beta=\frac{1}{C_{j}\left(L_{1}+L_{2}+2 K\right)}, \alpha=\frac{1}{R_{j} C_{j}}, a=\frac{1}{C_{j}}, x_{1}=\phi$, $x_{2}=V_{j}, I / C_{j}=x_{3}$ then we can rewrite eq. (28) as

$$
\begin{aligned}
& \dot{x_{1}}(t)=x_{2}(t) \\
& \dot{x_{2}}(t)=x_{3}(t)-a \sin \left(x_{1}(t)\right)-\alpha x_{2}(t) \\
& \dot{x_{3}}(t)=\beta\left(E-x_{2}(t)\right) .
\end{aligned}
$$

We couple $N$ such oscillators as above with a nearest neighbour coupling scheme via the junction voltage $\left(x_{2}\right)$ and periodic boundary conditions. In this case the third electrical state variable $I_{j}(t)$ is replaced by the sum of the currents flowing through the coils constituting the

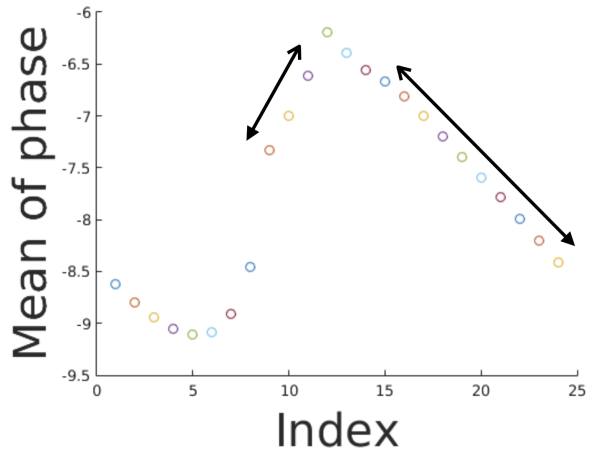

Figure 10. The splay phase-like structures are seen between oscillators 9 to 12 and 16 to 24 . The parameters are $\alpha=0.2$, $E=0.68, \beta=0.2, a=1, k=6$ with $N=24$ oscillators.

transformer. Using eq. (29) we can write the modified evolution equation for the $i$ th junction in such an array as

$$
\begin{aligned}
& \dot{x}_{1}{ }^{i}=x_{2}^{i} \\
& {\dot{x_{2}}}^{i}=x_{3}^{i}-a \sin \left(x_{1}^{i}\right)-(\alpha+2 k) x_{2}^{i}+k\left(x_{2}^{i+1}+x_{2}^{i-1}\right) \\
& \dot{x}_{3}{ }^{i}=\beta\left(E-x_{2}^{i}\right) .
\end{aligned}
$$

We indeed find splay-like structures (see figure 10) in this coupled system with a specific initial condition where $x_{1}^{i+1}-x_{1}^{i}=0.1, x_{2}^{i}=0, x_{3}^{i}=0.01$.

\section{Conclusion}

In this paper, we show that spatial splay states can exist in a bipartite coupled map lattice model consisting of identical sine circle map with different strength of intra and inter group coupling as well as in two groups of identical Josephson junctions with a similar coupling topology. We find that such states with their unique spatial configuration where the consecutive phase differences of the circle maps are constant, can only be realised using a special initial condition which is the single spatial splay state and at specific values of the system parameters. Figures $2 \mathrm{~d}$ and $6 \mathrm{a}$ suggest that the two copy splay states are quite robust with respect to the variation of the parameters $\Omega, \epsilon_{1}$ in our CML for $K$ values near zero. We also show the stability analysis of these states, both numerically and analytically using theorems from linear algebra and verify that the two copy spatial splay state is temporally chaotic in the CML. We find that this state bifurcates to the splay states with kinks, splay chimera states and globally synchronised states as $K$ increases to one.

The splay states can be observed in laboratory systems. Here we show that single spatial splay structure 
as well as two copy spatial splay structure can exist in a system of two arrays of Josephson junctions with similar global coupling schemes as the CML. We further show that the splay structures can also be observed in an array of Hartley-like oscillators with nearest neighbour coupling. We note that in this paper we concentrate mainly on the analysis of pure spatial splay states. The nature of the phase slipped splay states, splay states with kinks and the splay mixed states are yet to be explored. The splay states in the systems of eqs (27) and (30) also require rigorous analysis. We hope to study these in future.

\section{Acknowledgements}

JS thanks University Grants commission of India for the research fellowship. One of us (NG) thanks ICTPSAIFR for hospitality at the IFT (São Paulo) where this work was started as well as the grant CSIR 03(1294)/13/EMR-II. PL and HAC thank ICTP-SAIFR and FAPESP grant 2011/11973-4 for partial support. PL acknowledges support by FAPESP grant 2014/13272-1.

\section{References}

[1] C R Nayak and N Gupte, AIP Conf. Proc. 1339, 172 (2011)

[2] S Nkomo, M R Tinsley and K Showalter, Phys. Rev. Lett. 110, 244102 (2013)

[3] A M Hagerstrom, T E Murphy, R Roy, P Hövel, I Omelchenko and E Scholl, Nature Phys. 8, 658 (2012)
[4] K Y Tsang, R E Mirollo, S H Strogatz and K Wiesenfeld, Physica D 48, 102 (1991)

[5] K Wiesenfeld and J W Swift, Phys. Rev. E 51, 1020 (1995)

[6] S Nichols and K Wiesenfeld, Phys. Rev. E 50, 205 (1994)

[7] S H Strogatz and R E Mirollo, Phys. Rev. E 47, 220 (1993)

[8] M Calamai, A Politi and A Torcini, Phys. Rev. E 80, 036209 (2009)

[9] A A Selivanov, J Lehnert, T Dahms, P Hövel, A L Fradkov and E Schöll, Phys. Rev. E 85, 016201 (2012)

[10] J N Blakely, B R Reed, N J Corron, M T Stahl and K Myneni, Proc. SPIE 7669, Radar Sensor Technology XIV, 76690Q (April 23, 2010); doi:10.1117/12.850153.

[11] N F Rulkov, L Tsimring, M L Larsen and M Gabbay, Phys. Rev. E 74, 056205 (2006)

[12] D A Paley, N E Leonard, R Sepulchre, Oscillator models and collective motion: Splay state stabilization of self-propelled particles, in Proceedings of the 44th IEEE Conference on decision and control and European Control Conference 2005, Seville, Spain, December 12-15 (IEEE, Piscataway NJ, 2005)

[13] Y N Kyrychko, K B Blyuss and E Scholl, Chaos 24, 043117 (2014)

[14] A Nordenfelt, Chaos 25, 113110 (2015)

[15] M H Jensen, P Bak and T Bohr, Phys. Rev. Lett. 50, 1637 (1983)

[16] E Ott, Chaos in dynamical systems (Cambridge University Press, Cambridge, 1993)

[17] N Chatterjee and N Gupte, Phys. Rev. E 63, 017202 (2000)

[18] J Singha and N Gupte, Phys. Rev. E 94, 052204 (2016) 